\title{
Unbiased LambdaMART: An Unbiased Pairwise Learning-to-Rank Algorithm
}

\author{
Ziniu $\mathrm{Hu}^{*}$ \\ University of California, Los Angeles, USA \\ bull@cs.ucla.edu \\ Qu Peng \\ ByteDance AI Lab, Beijing, China \\ pengqu@bytedance.com
}

\author{
Yang Wang \\ ByteDance AI Lab, Beijing, China \\ wangyang.127@bytedance.com \\ Hang Li \\ ByteDance AI Lab, Beijing, China \\ lihang.lh@bytedance.com
}

\begin{abstract}
Recently a number of algorithms under the theme of 'unbiased learning-to-rank' have been proposed, which can reduce position bias, the major type of bias in click data, and train a high-performance ranker with click data. Most of the existing algorithms, based on the inverse propensity weighting (IPW) principle, first estimate the click bias at each position, and then train an unbiased ranker with the estimated biases using a learning-to-rank algorithm. However, there has not been a method for unbiased pairwise learning-to-rank that can simultaneously conduct debiasing of click data and training of a ranker using a pairwise loss function. In this paper, we propose a novel framework to accomplish the goal and apply this framework to the state-of-the-art pairwise learning-to-rank algorithm, LambdaMART. Our algorithm named Unbiased LambdaMART can jointly estimate the biases at click positions and the biases at unclick positions, and learn an unbiased ranker. Experiments on benchmark data show that Unbiased LambdaMART can significantly outperform existing algorithms by large margins. In addition, an online A/B Testing at a commercial search engine shows that Unbiased LambdaMART can effectively conduct debiasing of click data and enhance relevance ranking.
\end{abstract}

\section{CCS CONCEPTS}

- Information systems $\rightarrow$ Learning to rank.

\section{KEYWORDS}

Learning-to-Rank, Unbiased Learning-to-Rank, LambdaMART

\section{ACM Reference Format:}

Ziniu Hu, Yang Wang, Qu Peng, and Hang Li. 2019. Unbiased LambdaMART: An Unbiased Pairwise Learning-to-Rank Algorithm. In Proceedings of the 2019 World Wide Web Conference (WWW'19), May 13-17, 2019, San Francisco, CA, USA. ACM, New York, NY, USA, 7 pages. https://doi.org/10.1145/3308558. 3313447

${ }^{*}$ The full version of this paper is avaiable at arxiv. This work was done when the first author was an intern at ByteDance AI Lab.

This paper is published under the Creative Commons Attribution 4.0 International (CC-BY 4.0) license. Authors reserve their rights to disseminate the work on their personal and corporate Web sites with the appropriate attribution.

WWW'19, May 13-17, 2019, San Francisco, CA, USA

() 2019 IW3C2 (International World Wide Web Conference Committee), published under Creative Commons CC-BY 4.0 License.

ACM ISBN 978-1-4503-6674-8/19/05

https://doi.org/10.1145/3308558.3313447

\section{INTRODUCTION}

Learning-to-rank, which refers to machine learning techniques on automatically constructing a model (ranker) from data for ranking in search, has been widely used in current search systems. Existing algorithms can be categorized into pointwise, pairwise, and listwise approaches according to the loss functions they utilize [11-13]. Among the proposed algorithms, LambdaMART is a state-of-theart algorithm $[3,18]$. The data for training in learning-to-rank is usually labeled by human assessors so far, and the labelling process is often strenuous and costly. This raises the question of whether it is possible to train a ranker by using click data collected from the same search system. Click data is indicative of individual users' relevance judgments and is relatively easy to collect with low cost. On the other hand, it is also noisy and biased $[8,19]$. Notably, the orders of documents in the search results affect users' judgments. Users tend to more frequently click documents presented at higher positions, which is called position bias. This has been preventing practical use of click data in learning-to-rank.

Recently a new research direction, referred to as unbiased learningto-rank, is arising and making progress. Unbiased learning-to-rank aims at eliminating bias in click data, particularly position bias, and making use of the debiased data to train a ranker. Wang et al. [16] and Joachims et al. [9] respectively propose employing the inverse propensity weighting (IPW) principle [15] to learn an 'unbiased ranker' from click data. It is proved that the objective function in learning using IPW is an unbiased estimate of the risk function defined on a relevance measure (a pointwise loss). The authors also develop methods for estimating position bias by randomization of search results online. Wang et al. [17] further develop a method for estimating position bias from click data offline. More recently $\mathrm{Ai}$ et al. [1] propose a method that can jointly estimate position bias and train a ranker from click data, again on the basis of IPW. In the previous work, the IPW principle is limited to the pointwise setting in the sense that position biases are only defined on click positions.

In this paper, we address the problem of jointly estimating position biases and training a ranker from click data for pairwise learning-to-rank, particularly using a pairwise algorithm, LambdaMART. To do so, we extend the inverse propensity weighting principle to the pairwise setting, and develop a new method for jointly conducting position bias estimation and ranker training.

Specifically, we give a formulation of unbiased learning-to-rank for the pairwise setting and extend the IPW principle. We define position biases as the ratio of the click probability to the relevance 
probability at each position, as well as the ratio of the unclick probability to the irrelevance probability. This definition takes both the position biases at click positions and those at unclick positions into consideration. We prove that under the extended IPW principle, the objective function becomes an unbiased estimate of risk function defined on pairwise loss functions. In this way, we can learn an unbiased ranker using a pairwise ranking algorithm.

We then develop a method for jointly estimating position biases for both click and unclick positions and training a ranker for pairwise learning-to-rank, called Pairwise Debiasing. The position bias and the ranker can be iteratively learned through minimization of the same objective function. As an instance, we further develop Unbiased LambdaMART ${ }^{1}$, an algorithm of learning an unbiased ranker using LambdaMART.

Experiments on the Yahoo learning-to-rank challenge benchmark dataset demonstrate that Unbiased LambdaMART can effectively conduct debiasing of click data and significantly outperform the baseline algorithms in terms of all measures, for example, 3$4 \%$ improvements in terms of NDCG@1. An online A/B Testing at a commercial news search engine, Jinri Toutiao, also demonstrates that Unbiased LambdaMART can enhance the performance of relevance ranking at the search engine.

The contribution of this paper includes the following proposals.

- A general framework on unbiased learning-to-rank in the pairwise setting, particularly, an extended IPW.

- Pairwise Debiasing, a method for jointly estimating position bias and training a pairwise ranker.

- Unbiased LambdaMART, an algorithm of unbiased pairwise learning-to-rank using LambdaMART.

\section{RELATED WORK}

Learning-to-rank is to automatically construct a ranking model from data, referred to as a ranker, for ranking in search. The algorithms of learning-to-rank can be categorized as pointwise approach, pairwise approach, and listwise approach, based on the loss functions in learning [11-13]. The pairwise and listwise algorithms usually work better than the pointwise algorithms [12], because the key issue of ranking in search is to determine the orders of documents but not to judge the relevance of documents, which is exactly the goal of the pairwise and listwise algorithms.

Recently, a new direction in learning-to-rank, referred to as unbiased learning-to-rank, is arising and making progress. The goal of unbiased learning-to-rank is to develop new techniques to conduct debiasing of click data and leverage the debiased click data in training of a ranker[2].

Wang et al. [16] apply unbiased learning-to-rank to personal search. They conduct randomization of search results to estimate query-level position bias and adjust click data for training of a ranker in personal search on the basis of inverse propensity weighting (IPW) [15]. Joachims et al. [9] theoretically prove that with the inverse propensity weighting (IPW) principle, one can obtain an unbiased estimate of a risk function on relevance in learning-torank. They also utilize online randomization to estimate position bias and perform training of a RankSVM model. Wang et al. [17] employ a regression-based EM algorithm to infer position bias by

\footnotetext{
${ }^{1}$ Code is available at https://github.com/acbull/Unbiased_LambdaMart
}

maximizing the likelihood of click data. The estimated position bias is then utilized in learning of LambdaMART. Recently, Ai et al. [1] design a dual learning algorithm which can jointly learn an unbiased propensity model for representing position bias and an unbiased ranker for relevance ranking, by optimizing two objective functions. Both models are implemented as neural networks. Their method is also based on IPW, while the loss function is a pointwise loss function.

Our work mainly differs from the previous work in that:

- In previous work, position bias is defined as the observation probability, and thus IPW is limited to the pointwise setting. In this work, we give a more general definition of position bias, and extend IPW to the pairwise setting, in which the loss function is pairwise and debiasing is carried out at both click positions and unclick positions.

- In previous work, estimation of position bias either relies on randomization of search results online, which can hurt user experiences $[9,16]$, or resorts to separate learning of a propensity model from click data offline, which can be suboptimal to relevance ranking $[1,17]$. In this paper, we propose to jointly conduct estimation of position bias and learning of a ranker through minimizing one objective function.

\section{FRAMEWORK}

In this section, we give a general formulation of unbiased learningto-rank, for both the pointwise and pairwise settings, and extend the inverse propensity weighting principle to the pairwise setting.

\subsection{Pointwise Unbiased Learning-to-Rank}

In learning-to-rank, given a query document pair denoted as $x$, the ranker $f$ assigns a score to the document. The documents with respect to the query are then ranked in descending order of their scores. Traditionally, the ranker is learned with labeled data. In the pointwise setting, the loss function in learning is defined on a single data point $x$.

Let $q$ denote the query and $D_{q}$ the set of documents associated with $q$. Let $d_{i}$ denote the $i$-th document in $D_{q}$ and $x_{i}$ the feature vector of $q$ and $d_{i}$. Let $r_{i}^{+}$and $r_{i}^{-}$represent that $d_{i}$ is relevant and irrelevant respectively. The risk function in learning is defined as

$$
R_{r e l}(f)=\int L\left(f\left(x_{i}\right), r_{i}^{+}\right) d P\left(x_{i}, r_{i}^{+}\right)
$$

where $f$ denotes a ranker, $L\left(f\left(x_{i}\right), r_{i}^{+}\right)$denotes a pointwise loss function based on an IR measure [9] and $P\left(x_{i}, r_{i}^{+}\right)$denotes the probability distribution on $x_{i}$ and $r_{i}^{+}$.

Suppose that there is a labeled dataset in which the relevance of documents with respect to queries is given. One can learn a ranker $\hat{f}_{\text {rel }}$ through the minimization of the empirical risk function (objective function) as follows.

$$
\hat{f}_{\text {rel }}=\arg \min _{f} \sum_{q} \sum_{d_{i} \in D_{q}} L\left(f\left(x_{i}\right), r_{i}^{+}\right)
$$

One can also consider using click data as relevance feedbacks from users, more specifically, viewing clicked documents as relevant documents and unclicked documents as irrelevant documents, and training a ranker with a click dataset. This is what we call 'biased learning-to-rank', because click data has position bias, presentation 
bias, etc. For convenience, let us assume that document $d_{i}$ in $D_{q}$ is exactly the document ranked at position $i$ by the original ranker. Let $c_{i}^{+}$and $c_{i}^{-}$represent that document $d_{i}$ is clicked and unclicked in the click dataset respectively. The risk function and minimization of empirical risk function can be defined as follows.

$$
\begin{aligned}
R_{\text {click }}(f) & =\int L\left(f\left(x_{i}\right), c_{i}^{+}\right) d P\left(x_{i}, c_{i}^{+}\right) \\
\hat{f}_{\text {click }} & =\arg \min _{f} \sum_{q} \sum_{d_{i} \in D_{q}} L\left(f\left(x_{i}\right), c_{i}^{+}\right)
\end{aligned}
$$

The loss function is defined on clicked documents with label $c_{i}^{+}$. The ranker $\hat{f}_{\text {click }}$ learned in this way is biased, however.

Unbiased learning-to-rank aims to eliminate the biases, for example position bias, in the click data and train a ranker with the debiased data. The training of ranker and debiasing of click data can be performed simultaneously or separately. The key question is how to fill the gap between click and relevance, that is, $P\left(c_{i}^{+} \mid x_{i}\right)$ and $P\left(r_{i}^{+} \mid x_{i}\right)$. Here we assume that the click probability is proportional to the relevance probability at each position, where the ratio $t_{i}^{+}>0$ is referred to as bias at a click position $i$.

$$
P\left(c_{i}^{+} \mid x_{i}\right)=t_{i}^{+} P\left(r_{i}^{+} \mid x_{i}\right)
$$

There are $k$ ratios corresponding to $k$ positions. The ratios can be affected by different types of bias, but in this paper, we only consider position bias.

We can conduct learning of an unbiased ranker $\hat{f}_{\text {unbiased }}$, through minimization of the empirical risk function as follows.

$$
\begin{aligned}
R_{\text {unbiased }}(f) & =\int \frac{L\left(f\left(x_{i}\right), c_{i}^{+}\right)}{t_{i}^{+}} d P\left(x_{i}, c_{i}^{+}\right) \\
& =\int \frac{L\left(f\left(x_{i}\right), c_{i}^{+}\right)}{\frac{P\left(c_{i}^{+} \mid x_{i}\right)}{P\left(r_{i}^{+} \mid x_{i}\right)}} d P\left(x_{i}, c_{i}^{+}\right) \\
& =\int L\left(f\left(x_{i}\right), c_{i}^{+}\right) d P\left(x_{i}, r_{i}^{+}\right) \\
& =\int L\left(f\left(x_{i}\right), r_{i}^{+}\right) d P\left(x_{i}, r_{i}^{+}\right)=R_{r e l}(f) \\
\hat{f}_{\text {unbiased }} & =\arg \min _{f} \sum_{q} \sum_{d_{i} \in D_{q}} \frac{L\left(f\left(x_{i}\right), c_{i}^{+}\right)}{t_{i}^{+}}
\end{aligned}
$$

In (9) click label $c_{i}^{+}$in the loss function is replaced with relevance label $r_{i}^{+}$, because after debiasing click implies relevance.

One justification of this method is that $R_{\text {unbiased }}$ is in fact an unbiased estimate of $R_{r e l}$. This is the so-called inverse propensity weighting (IPW) principle proposed in previous work. That is to say, if we can properly estimate position bias (ratio) $t_{i}^{+}$, then we can reliably train an unbiased ranker $\hat{f}_{\text {unbiased }}$.

\subsection{Pairwise Unbiased Learning-to-Rank}

In the pairwise setting, the ranker $f$ is still defined on a query document pair $x$, and the loss function is defined on two data points $x_{i}$ and $x_{j}$. Traditionally, the ranker is learned with labeled data.

Let $q$ denote a query. Let $d_{i}$ and $d_{j}$ denote the $i$-th and $j$-th documents with respect to query $q$. Let $x_{i}$ and $x_{j}$ denote the feature vectors from $d_{i}$ and $d_{j}$ as well as $q$. Let $r_{i}^{+}$and $r_{j}^{-}$represent that document $d_{i}$ and document $d_{j}$ are relevant and irrelevant respectively. Let $I_{q}$ denote the set of document pairs $\left(d_{i}, d_{j}\right)$ where $d_{i}$ is relevant and $d_{j}$ is irrelevant. For simplicity we only consider binary relevance here and one can easily extend it to the multi-level relevance case. The risk function and the minimization of empirical risk function are defined as

$$
\begin{aligned}
R_{r e l}(f) & =\int L\left(f\left(x_{i}\right), r_{i}^{+}, f\left(x_{j}\right), r_{j}^{-}\right) d P\left(x_{i}, r_{i}^{+}, x_{j}, r_{j}^{-}\right) \\
\hat{f}_{r e l} & =\arg \min _{f} \sum_{q} \sum_{\left(d_{i}, d_{j}\right) \in I_{q}} L\left(f\left(x_{i}\right), r_{i}^{+}, f\left(x_{j}\right), r_{j}^{-}\right)
\end{aligned}
$$

where $L\left(f\left(x_{i}\right), r_{i}^{+}, f\left(x_{j}\right), r_{j}^{-}\right)$denotes a pairwise loss function.

One can consider using click data to directly train a ranker, that is, to conduct 'biased learning-to-rank'. Let $c_{i}^{+}$and $c_{j}^{-}$represent that document $d_{i}$ and document $d_{j}$ are clicked and unclicked respectively. Let $I_{q}$ denote the set of document pairs $\left(d_{i}, d_{j}\right)$ where $d_{i}$ is clicked and $d_{j}$ is unclicked. The risk function and minimization of empirical risk function can be defined as follows.

$$
\begin{aligned}
R_{\text {click }}(f) & =\int L\left(f\left(x_{i}\right), c_{i}^{+}, f\left(x_{j}\right), c_{j}^{-}\right) d P\left(x_{i}, c_{i}^{+}, x_{j}, c_{j}^{-}\right) \\
\hat{f}_{\text {click }} & =\arg \min _{f} \sum_{q} \sum_{\left(d_{i}, d_{j}\right) \in I_{q}} L\left(f\left(x_{i}\right), c_{i}^{+}, f\left(x_{j}\right), c_{j}^{-}\right)
\end{aligned}
$$

The ranker $\hat{f}_{\text {click }}$ is however biased.

Similar to the pointwise setting, we consider dealing with position bias in the pairwise setting and assume that the click probability is proportional to the relevance probability at each position and the unclick probability is proportional to the irrelevance probability at each position. The ratios $t_{i}^{+}>0$ and $t_{j}^{-}>0$ are referred to as position biases at a click position $i$ and an unclick position $j$.

$$
\begin{aligned}
& P\left(c_{i}^{+} \mid x_{i}\right)=t_{i}^{+} P\left(r_{i}^{+} \mid x_{i}\right) \\
& P\left(c_{j}^{-} \mid x_{j}\right)=t_{j}^{-} P\left(r_{i}^{-} \mid x_{j}\right)
\end{aligned}
$$

There are $2 k$ position biases (ratios) corresponding to $k$ positions.

We can conduct learning of an unbiased ranker $\hat{f}_{\text {unbiased }}$, through minimization of the empirical risk function as follows.

$$
\begin{aligned}
R_{\text {unbiased }}(f) & =\int \frac{L\left(f\left(x_{i}\right), c_{i}^{+}, f\left(x_{j}\right), c_{j}^{-}\right)}{t_{i}^{+} \cdot t_{j}^{-}} d P\left(x_{i}, c_{i}^{+}, x_{j}, c_{j}^{-}\right) \\
& =\iint \frac{L\left(f\left(x_{i}\right), c_{i}^{+}, f\left(x_{j}\right), c_{j}^{-}\right) d P\left(c_{i}^{+}, x_{i}\right) d P\left(c_{j}^{-}, x_{j}\right)}{\frac{P\left(c_{i}^{+} \mid x_{i}\right) P\left(c_{j}^{-} \mid x_{j}\right)}{P\left(r_{i}^{+} \mid x_{i}\right) P\left(r_{i}^{-} \mid x_{j}\right)}} \\
& =\iint L\left(f\left(x_{i}\right), c_{i}^{+}, f\left(x_{j}\right), c_{j}^{-}\right) d P\left(r_{i}^{+}, x_{i}\right) d P\left(r_{i}^{-}, x_{j}\right) \\
& =\int L\left(f\left(x_{i}\right), r_{i}^{+}, f\left(x_{j}\right), r_{j}^{-}\right) d P\left(x_{i}, r_{i}^{+}, x_{j}, r_{j}^{-}\right) \\
& =R_{r e l}(f) \\
\hat{f}_{\text {unbiased }} & =\arg \min _{f} \sum_{q} \sum_{\left(d_{i}, d_{j}\right) \in I_{q}} \frac{L\left(f\left(x_{i}\right), c_{i}^{+}, f\left(x_{j}\right), c_{j}^{-}\right)}{t_{i}^{+} \cdot t_{j}^{-}}
\end{aligned}
$$

In (17) it is assumed that relevance and click at position $i$ are independent from those at position $j$. In (20), click labels $c_{i}^{+}$and $c_{j}^{-}$ 
are replaced with relevance labels $r_{i}^{+}$and $r_{j}^{-}$because after debiasing click implies relevance and unclick implies irrelevance.

Similar to the pointwise setting, $R_{\text {unbiased }}$ is an unbiased estimate of $R_{\text {rel }}$. Therefore, if we can accurately estimate the position bias (ratios) $t_{i}^{+}$and $t_{j}^{-}$, then we can reliably train an unbiased ranker $\hat{f}_{\text {unbiased }}$. This is an extension of the inverse propensity weighting (IPW) principle to the pairwise setting.

\section{APPROACH}

In this section, we present Pairwise Debiasing as a method of jointly estimating position bias and training a ranker for unbiased pairwise learning-to-rank. Furthermore, we apply Pairwise Debiasing on LambdaMart and describe the learning algorithm of Unbiased LambdaMART.

\subsection{Learning Strategy}

We first give a general strategy for pairwise unbiased learning-torank, named Pairwise Debiasing.

A key issue of unbiased learning-to-rank is to accurately estimate position bias. Previous work either relies on randomization of search results online, or resorts to a separate learning of position bias from click data offline. In this paper, we propose to simultaneously conduct estimation of position bias and learning of a ranker offline through minimizing the following regularized loss function.

$$
\begin{aligned}
& \min _{f, t^{+}, t^{-}} \mathcal{L}\left(f, t^{+}, t^{-}\right) \\
= & \min _{f, t^{+}, t^{-}} \sum_{q} \sum_{\left(d_{i}, d_{j}\right) \in I_{q}} \frac{L\left(f\left(x_{i}\right), c_{i}^{+}, f\left(x_{j}\right), c_{j}^{-}\right)}{t_{i}^{+} \cdot t_{j}^{-}}+\left\|t^{+}\right\|_{p}^{p}+\left\|t^{-}\right\|_{p}^{p} \\
& \text { s.t. } t_{1}^{+}=1, t_{1}^{-}=1
\end{aligned}
$$

where $f$ denotes a ranker, $t^{+}$and $t^{-}$denote position biases (ratios) at all positions, $L$ denotes a pairwise loss function, $\|\cdot\|_{p}^{p}$ denotes $L_{p}$ regularization. Because the position biases are relative values with respect to positions, to simplify the optimization process we fix the position biases of the first position to 1 and only learn the (relative) position biases of the rest of the positions. Here $p \in$ $[0,+\infty)$ is a hyper-parameter. The higher the value of $p$ is, the more regularization we impose on the position biases.

In the objective function, the position biases $t^{+}$and $t^{-}$are inversely proportional to the pairwise loss $L\left(f\left(x_{i}\right), c_{i}^{+}, f\left(x_{j}\right), c_{j}^{-}\right)$, and thus the estimated position biases will be high if the losses on those pairs of positions are high in the minimization. The position biases are regularized and constrained to avoid a trivial solution of infinity

It would be difficult to directly optimize the objective function in (24). We adopt a greedy approach to perform the task. Specifically, for the three optimization variables $f, t^{+}, t^{-}$, we iteratively optimize the objective function $\mathcal{L}$ with respect to one of them with the others fixed; we repeat the process until convergence.

\subsection{Estimation of position bias ratios}

Given a fixed ranker, we can estimate the position biases at all positions. There are in fact closed form solutions for the estimation.
The partial derivative of objective function $\mathcal{L}$ with respect to position bias $t^{+}$is

$\frac{\partial \mathcal{L}\left(f^{*}, t^{+},\left(t^{-}\right)^{*}\right)}{\partial t_{i}^{+}}=\sum_{q} \sum_{j:\left(d_{i}, d_{j}\right) \in I_{q}} \frac{L\left(f^{*}\left(x_{i}\right), c_{i}^{+}, f^{*}\left(x_{j}\right), c_{j}^{-}\right)}{-\left(t_{i}^{+}\right)^{2} \cdot\left(t_{j}^{-}\right)^{*}}+p \cdot\left(t_{i}^{+}\right)^{p-1}$

Thus, we have

$$
\begin{gathered}
\arg \min _{t_{i}^{+}} \mathcal{L}\left(f^{*}, t^{+},\left(t^{-}\right)^{*}\right)=\left[\sum_{q} \sum_{j:\left(d_{i}, d_{j}\right) \in I_{q}} \frac{L\left(f^{*}\left(x_{i}\right), c_{i}^{+}, f^{*}\left(x_{j}\right), c_{j}^{-}\right)}{p \cdot\left(t_{j}^{-}\right)^{*}}\right]^{\frac{1}{p+1}} \\
t_{i}^{+}=\left[\frac{\sum_{q} \sum_{j:\left(d_{i}, d_{j}\right) \in I_{q}}\left(L\left(f^{*}\left(x_{i}\right), c_{i}^{+}, f^{*}\left(x_{j}\right), c_{j}^{-}\right) /\left(t_{j}^{-}\right)^{*}\right)}{\sum_{q} \sum_{k:\left(d_{1}, d_{k}\right) \in I_{q}}\left(L\left(f^{*}\left(x_{1}\right), c_{1}^{+}, f^{*}\left(x_{k}\right), c_{k}^{-}\right) /\left(t_{k}^{-}\right)^{*}\right)}\right]^{\frac{1}{p+1}}
\end{gathered}
$$

In (28) the result is normalized to make the position bias at the first position to be 1 .

Similarly, we have

$$
t_{j}^{-}=\left[\frac{\sum_{q} \sum_{i:\left(d_{i}, d_{j}\right) \in I_{q}}\left(L\left(f^{*}\left(x_{i}\right), c_{i}^{+}, f^{*}\left(x_{j}\right), c_{j}^{-}\right) /\left(t_{i}^{+}\right)^{*}\right)}{\sum_{q} \sum_{k:\left(d_{k}, d_{1}\right) \in I_{q}}\left(L\left(f^{*}\left(x_{k}\right), c_{k}^{+}, f^{*}\left(x_{1}\right), c_{1}^{-}\right) /\left(t_{k}^{+}\right)^{*}\right)}\right]^{\frac{1}{p+1}}
$$

In this way, we can estimate the position biases (ratios) $t^{+}$and $t^{-}$in one step given a fixed ranker $f^{*}$. Note that the method here, referred to as Pairwise Debiasing, can be applied to any pairwise loss function. In this paper, we choose to apply the pairwise learning-to-rank algorithm LambdaMART.

\subsection{Learning of Ranker}

Given fixed position biases, we can learn an unbiased ranker. The partial derivative of $\mathcal{L}$ with respect to $f$ can be written in the following general form.

$\frac{\partial \mathcal{L}\left(f,\left(t^{+}\right)^{*},\left(t^{-}\right)^{*}\right)}{\partial f}=\sum_{q} \sum_{\left(d_{i}, d_{j}\right) \in I_{q}} \frac{1}{\left(t_{i}^{+}\right)^{*} \cdot\left(t_{j}^{-}\right)^{*}} \frac{\partial L\left(f\left(x_{i}\right), c_{i}^{+}, f\left(x_{j}\right), c_{j}^{-}\right)}{\partial f}$

We employ LambdaMART to train a ranker. LambdaMART [4, 18] employs gradient boosting or MART [6] and the gradient function of the loss function called lambda function. Given training data, it performs minimization of the objective function using the lambda function.

In LambdaMART, the lambda gradient $\lambda_{i}$ of document $d_{i}$ is calculated using all pairs of the other documents with respect to the query.

$$
\begin{gathered}
\lambda_{i}=\sum_{j:\left(d_{i}, d_{j}\right) \in I_{q}} \lambda_{i j}-\sum_{j:\left(d_{j}, d_{i}\right) \in I_{q}} \lambda_{j i} \\
\lambda_{i j}=\frac{-\sigma}{1+e^{\sigma\left(f\left(x_{i}\right)-f\left(x_{j}\right)\right)}}\left|\Delta Z_{i j}\right|
\end{gathered}
$$

where $\lambda_{i j}$ is the lambda gradient defined on a pair of documents $d_{i}$ and $d_{j}, \sigma$ is a constant with a default value of $2, f\left(x_{i}\right)$ and $f\left(x_{j}\right)$ are the scores of the two documents given by LambdaMART, $\Delta Z_{i j}$ 
denotes the difference between NDCG[7] scores if documents $d_{i}$ and $d_{j}$ are swapped in the ranking list.

Following the discussion above, we can make an adjustment on the lambda gradient $\tilde{\lambda}_{i}$ with the estimated position biases:

$$
\tilde{\lambda}_{i}=\sum_{j:\left(d_{i}, d_{j}\right) \in I_{q}} \frac{\lambda_{i j}}{\left(t_{i}^{+}\right)^{*} \cdot\left(t_{j}^{-}\right)^{*}}-\sum_{k:\left(d_{k}, d_{i}\right) \in I_{q}} \frac{\lambda_{k i}}{\left(t_{k}^{+}\right)^{*} \cdot\left(t_{i}^{-}\right)^{*}}
$$

Thus, by simply replacing the lambda gradient $\lambda_{i}$ in LambdaMART with the adjusted lambda gradient $\tilde{\lambda}_{i}$, we can reliably learn an unbiased ranker with the LambdaMART algorithm. We call the algorithm Unbiased LambdaMART.

\subsection{Learning Algorithm}

The learning algorithm of Unbiased LambdaMART is given in Algorithm 1 . The input is a click dataset $\mathcal{D}$. The hyper-parameters are regularization parameter $p$ and total number of boosting iterations $M$. The output is an unbiased ranker $f$ and estimated position biases at all positions $t^{+}, t^{-}$. As is outlined in Algorithm 1, Unbiased LambdaMART iteratively calculates adjusted lambda gradient in line 4, re-trains a ranker with the gradients in line 6, and re-estimates position biases in line 7 .

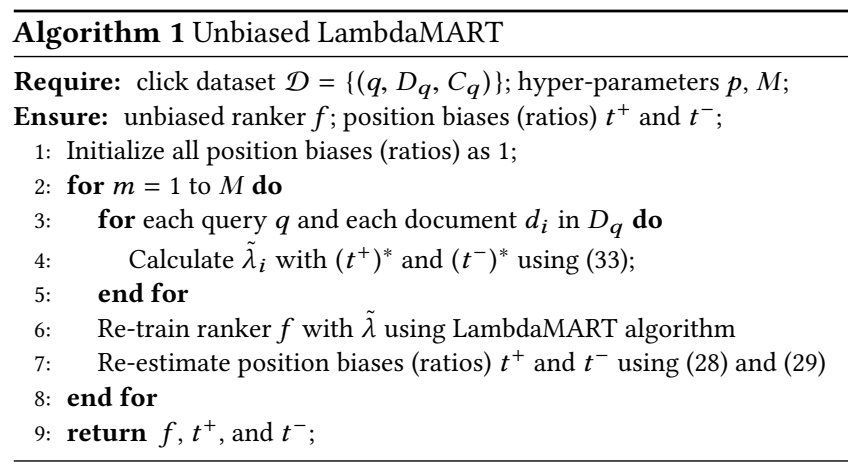

\section{EXPERIMENTS}

In this section, we present the results of two experiments on our proposed algorithm Unbiased LambdaMART. One is an offline experiment on a benchmark dataset. The other experiment is an online $\mathrm{A} / \mathrm{B}$ testing at a commercial search engine.

\subsection{Experiment on Benchmark Data}

We made use of the Yahoo! learning-to-rank challenge dataset ${ }^{1}$ to conduct an experiment. The Yahoo dataset is one of the largest benckmark dataset for learning-to-rank. It consists of 29921 queries and 710k documents. Each query document pair is represented by a 700-dimensional feature vector manually assigned with a label denoting relevance at 5 levels [5].

There is no click data associated with the Yahoo dataset. We followed the procedure in [1] to generate synthetically click data from the Yahoo dataset for offline evaluation.

\footnotetext{
${ }^{1}$ http://webscope.sandbox.yahoo.com
}

5.1.1 Click Data Generation. The click data generation process in [1] is as follows. First, one trains a Ranking SVM model using $1 \%$ of the training data with relevance labels, and uses the model to create an initial ranking list for each query. Next, one samples clicks from the ranking lists by simulating the browsing process of users. The position-based click model (PBM) [14] is utilized. It assumes that a user decides to click a document according to probability $P\left(c_{i}^{+}\right)=P\left(o_{i}^{+}\right) P\left(r_{i}^{+}\right)$. Here $P\left(o_{i}^{+}\right)$and $P\left(r_{i}^{+}\right)$are the observation probability and relevance probability respectively.

The probability of observation $P\left(o_{i}^{+}\right)$is calculated by

$$
P\left(o_{i}^{+} \mid x_{i}\right)=\rho_{i}^{\theta}
$$

where $\rho_{i}$ represents position bias at position $i$ and $\theta \in[0,+\infty]$ is a parameter controlling the degree of position bias. The position bias $\rho_{i}$ is obtained from an eye-tracking experiment in [8] and the parameter $\theta$ is set as 1 by default.

The probability of relevance $P\left(r_{i}^{+}\right)$is calculated by

$$
P\left(r_{i}^{+}\right)=\epsilon+(1-\epsilon) \frac{2^{y}-1}{2^{y \max }-1}
$$

where $y \in[0,4]$ represents a relevance level. The parameter $\epsilon$ represents click noise, which is set as 0.1 by default.

5.1.2 Baseline Methods. We made comprehensive comparisons between our method and the baselines. The baselines were created by combining the state-of-the-art debiasing methods and learningto-rank algorithms. There were six debiasing methods. To make fair comparison, we used click model to generate 165660 query sessions as training dataset, and utilized the same dataset for all debiasing methods. All the hyper-parameters of the baseline models were the same as those in the original papers.

Randomization: The method, proposed by Joachims et al. [9], uses randomization to infer the observation probabilities as position biases. We randomly shuffled the rank lists and then estimated the position biases as in [1].

Regression-EM: The method, proposed by Wang et al. [17], directly estimates the position biases using a regression-EM model implemented by GBDT.

Dual Learning Algorithm: The method, proposed by Ai et al. [1], jointly learns a ranker and conducts debiasing of click data. The algorithm implements both the ranking model and the debiasing model as deep neural networks.

Pairwise Debiasing: Our proposed debiasing method, which is combined with LambdaMART. In this experiment, we set the hyper-parameter $p$ as 0 by default. As explained below, a further experiment was conducted with different values of $p$.

Click Data: In this method, the raw click data without debiasing is used to train a ranker, whose performance is considered as a lower bound.

Labeled Data: In this method, human annotated relevance labels without any bias are used as data for training of ranker, whose performance is considered as an upper bound.

There were three learning-to-rank algorithms.

DNN: A deep neural network was implemented as a ranker, as in [1]. We directly used the code provided by Ai et al. ${ }^{2}$.

\footnotetext{
${ }^{2}$ https://github.com/QingyaoAi/Dual-Learning-Algorithm-for-Unbiased-Learning-to-Rank
} 
Table 1: Comparison of different unbiased learning-to-rank methods.

\begin{tabular}{c|c||c|c|c|c|c}
\hline Ranker & Debiasing Method & MAP & NDCG@1 & NDCG@3 & NDCG@5 & NDCG@10 \\
\hline \hline \multirow{4}{*}{ LambdaMART } & Labeled Data (Upper Bound) & 0.854 & 0.745 & 0.745 & 0.757 & 0.790 \\
\cline { 2 - 6 } & Pairwise Debiasing & $\mathbf{0 . 8 3 6}$ & $\mathbf{0 . 7 1 7}$ & $\mathbf{0 . 7 1 6}$ & $\mathbf{0 . 7 2 8}$ & $\mathbf{0 . 7 6 4}$ \\
\cline { 2 - 7 } & Regression-EM [17] & 0.830 & 0.685 & 0.684 & 0.700 & 0.743 \\
\cline { 2 - 7 } & Randomization & 0.827 & 0.669 & 0.678 & 0.690 & 0.728 \\
\cline { 2 - 7 } & Click Data (Lower Bound) & 0.820 & 0.658 & 0.669 & 0.672 & 0.716 \\
\hline \hline \multirow{5}{*}{ DNN } & Labeled Data (Upper Bound) & 0.831 & 0.677 & 0.685 & 0.705 & 0.737 \\
\cline { 2 - 7 } & Dual Learning Algorithm [1] & 0.828 & 0.674 & 0.683 & 0.697 & 0.734 \\
\cline { 2 - 7 } & Regression-EM & 0.829 & 0.676 & 0.684 & 0.699 & 0.736 \\
\cline { 2 - 7 } & Randomization & 0.825 & 0.673 & 0.679 & 0.693 & 0.732 \\
\cline { 2 - 7 } & Click Data (Lower Bound) & 0.819 & 0.637 & 0.651 & 0.667 & 0.711 \\
\hline \hline \multirow{5}{*}{ RankSVM } & Labeled Data (Upper Bound) & 0.815 & 0.631 & 0.649 & 0.675 & 0.707 \\
\cline { 2 - 7 } & Regression-EM & 0.815 & 0.629 & 0.648 & 0.674 & 0.705 \\
\cline { 2 - 7 } & Randomization [9] & 0.814 & 0.628 & 0.644 & 0.672 & 0.707 \\
\cline { 2 - 7 } & Click Data (Lower Bound) & 0.811 & 0.614 & 0.629 & 0.658 & 0.697 \\
\hline
\end{tabular}

RankSVM: We directly used the Unbiased RankSVM Software provided by Joachims et al. ${ }^{3}$, with hyper-parameter $C$ being 200 .

LambdaMART: We implemented Unbiased LambdaMART by modifying the LambdaMART tool in LightGBM [10]. We utilized the default hyper-parameters of the tool. The total number of trees was 300 , learning rate was 0.05 , number of leaves for one tree was 31 , feature fraction was 0.9 , and bagging fraction was 0.9 .

In summary, there were 13 baselines to compare with our proposed Unbiased LambdaMART algorithm. Note that Dual Learning Algorithm and DNN are tightly coupled. We did not combine Pairwise Debiasing with RankSVM and DNN, as it is beyond the scope of this paper.

5.1.3 Experimental Results. Table 1 summarizes the results. We can see that our method of Unbiased LambdaMART (LambdaMART + Pairwise Debiasing) significantly outperforms all the other baseline methods. The results of Regression-EM and Dual Learning Algorithm are compariable with those reported in the original papers. In particular, we have the following findings.

- Our method of LambdaMART+Pairwise Debiasing (Unbiased LambdaMART) achieves better performances than all the state-of-the-art methods in terms of all measures. For example, in terms of NDCG@1, our method outperforms LambdaMART+Regression-EM by 3.2\%, outperforms DNN+Dual Learning by $4.3 \%$, and outperforms RankSVM+Randomization by $8.9 \%$.

- Pairwise Debiasing works better than the other debiasing methods. When combined with LambdaMART, Pairwise Debiasing outperforms Regression-EM by $3.2 \%$, outperforms Randomization by $4.8 \%$ in terms of NDCG@1.

- LambdaMART trained with human labeled data achieves the best performance (upper bound). An unbiased learning-torank algorithm can still not beat it. This indicates that there is still room for improvement in unbiased learning-to-rank.

\footnotetext{
${ }^{3}$ https://www.cs.cornell.edu/people/tj/svm_light/svm_proprank.html
}

Table 2: Relative increases of first click ratios by Unbiased LambdaMART in online $\mathrm{A} / \mathrm{B}$ testing.

\begin{tabular}{c|c|c|c}
\hline Measure & Click@1 & Click@3 & Click@5 \\
\hline \hline Increase & $2.64 \%$ & $1.21 \%$ & $0.80 \%$ \\
\hline P-value & 0.001 & 0.004 & 0.023 \\
\hline
\end{tabular}

\subsection{A/B Testing at Commercial Search Engine}

We further evaluated the performance of Unbiased LambdaMART by deploying it at the search engine of Jinri Toutiao, a commercial news recommendation app in China with over 100 million daily active users. We trained two rankers with Unbiased LambdaMART and LambdaMART + Click Data using click data of approximately 19.6 million query sessions collected over two days at the search engine. Then we deployed the two rankers at the search system to conduct $\mathrm{A} / \mathrm{B}$ testing. The $\mathrm{A} / \mathrm{B}$ testing was carried out for 16 days. In each experiment group, the ranker was randomly assigned approximately 1.5 million queries per day.

In the online environment, we observed that different users have quite different click behaviors. It appeared to be necessary to have a tighter control on debiasing. We therefore set the hyper-parameter $p$ as 1 , i.e., we conducted $L_{1}$ regularization to impose a stronger regularization on the position biases. We validated the correctness of this hyper-parameter selection on a small set of relevance dataset.

We compared the results of the two rankers in terms of first click ratios, which are the percentages of sessions having first clicks at top 1,3,5 positions among all sessions. A ranker with higher first click ratios should have better performance.

As shown in Table 2, Unbiased LambdaMART can significantly outperform LambdaMART + Click Data in terms of first click ratios at the $\mathrm{A} / \mathrm{B}$ Testing. It increases the first click ratios at positions $1,3,5$ by $2.64 \%, 1.21 \%$ and $0.80 \%$, respectively, which are all statistically significant ( $p$-values $<0.05$ ). It indicates that Unbiased LambdaMART can make significantly better relevance ranking. 


\section{CONCLUSION}

In this paper, we have proposed a general framework for pairwise unbiased learning-to-rank, including the extended inverse propensity weighting (IPW) principle. We have also proposed a method called Pairwise Debiasing to jointly estimate position biases and train a ranker by directly optimizing a same objective function within the framework. We develop a new algorithm called Unbiased LambdaMART as application of the method. Experimental results show that Unbiased LambdaMART achieves significantly better results than the existing methods on a benchmark dataset, and is effective in relevance ranking at a real-world search system.

\section{REFERENCES}

[1] Qingyao Ai, Keping Bi, Cheng Luo, Jiafeng Guo, and W. Bruce Croft. 2018. Unbiased Learning to Rank with Unbiased Propensity Estimation. In The 41st International ACM SIGIR Conference on Research \& Development in Information Retrieval, SIGIR 2018, Ann Arbor, MI, USA, July 08-12, 2018. 385-394.

[2] Qingyao Ai, Jiaxin Mao, Yiqun Liu, and W. Bruce Croft. 2018. Unbiased Learning to Rank: Theory and Practice. In Proceedings of the 27th ACM International Conference on Information and Knowledge Management, CIKM 2018. ACM, 2305-2306.

[3] Chris J.C. Burges. 2010. From RankNet to LambdaRank to LambdaMART: An Overview. Technical Report.

[4] Christopher J. C. Burges, Robert Ragno, and Quoc Viet Le. 2006. Learning to Rank with Nonsmooth Cost Functions. In Proceedings of the 20th Annual Conference on Neural Information Processing Systems, NIPS 2006, Vancouver, British Columbia, Canada, December 4-7, 2006. 193-200.

[5] Olivier Chapelle and Yi Chang. 2011. Yahoo! Learning to Rank Challenge Overview. In Proceedings of the Yahoo! Learning to Rank Challenge, held at ICML 2010, Haifa, Israel, June 25, 2010. 1-24

[6] Jerome H. Friedman. 2000. Greedy Function Approximation: A Gradient Boosting Machine. Annals of Statistics 29 (2000), 1189-1232.

[7] Kalervo Järvelin and Jaana Kekäläinen. 2002. Cumulated gain-based evaluation of IR techniques. ACM Trans. Inf. Syst. 20, 4 (2002), 422-446.

[8] Thorsten Joachims, Laura A. Granka, Bing Pan, Helene Hembrooke, and Geri Gay. 2005. Accurately interpreting clickthrough data as implicit feedback. In
SIGIR 2005: Proceedings of the 28th Annual International ACM SIGIR Conference on Research and Development in Information Retrieval, Salvador, Brazil, August 15-19, 2005. 154-161.

[9] Thorsten Joachims, Adith Swaminathan, and Tobias Schnabel. 2017. Unbiased Learning-to-Rank with Biased Feedback. In Proceedings of the Tenth ACM International Conference on Web Search and Data Mining, WSDM 2017, Cambridge, United Kingdom, February 6-10, 2017. 781-789.

[10] Guolin Ke, Qi Meng, Thomas Finley, Taifeng Wang, Wei Chen, Weidong Ma, Qiwei Ye, and Tie-Yan Liu. 2017. LightGBM: A Highly Efficient Gradient Boosting Decision Tree. In Advances in Neural Information Processing Systems 30: Annual Conference on Neural Information Processing Systems, NIPS 2017, 4-9 December 2017, Long Beach, CA, USA. 3149-3157.

[11] Hang Li. 2011. A Short Introduction to Learning to Rank. IEICE Transactions 94-D, 10 (2011), 1854-1862.

[12] Hang Li. 2014. Learning to Rank for Information Retrieval and Natural Language Processing, Second Edition. Morgan \& Claypool Publishers.

[13] Tie-Yan Liu. 2009. Learning to Rank for Information Retrieval. Foundations and Trends in Information Retrieval 3, 3 (2009), 225-331.

[14] Matthew Richardson, Ewa Dominowska, and Robert Ragno. 2007. Predicting clicks: estimating the click-through rate for new ads. In Proceedings of the 16th International Conference on World Wide Web, WWW 2007, Banff, Alberta, Canada, May 8-12, 2007. 521-530.

[15] Paul R. Rosenbaum and Donald B. Rubin. 1983. The Central Role of the Propensity Score in Observational Studies for Causal Effects. Biometrika 70 (1983), 41-55.

[16] Xuanhui Wang, Michael Bendersky, Donald Metzler, and Marc Najork. 2016. Learning to Rank with Selection Bias in Personal Search. In Proceedings of the 39th International ACM SIGIR conference on Research and Development in Information Retrieval, SIGIR 2016, Pisa, Italy, fuly 17-21, 2016. 115-124.

[17] Xuanhui Wang, Nadav Golbandi, Michael Bendersky, Donald Metzler, and Marc Najork. 2018. Position Bias Estimation for Unbiased Learning to Rank in Personal Search. In Proceedings of the Eleventh ACM International Conference on Web Search and Data Mining, WSDM 2018, Marina Del Rey, CA, USA, February 5-9, 2018. 610-618.

[18] Qiang Wu, Christopher J. C. Burges, Krysta Marie Svore, and Jianfeng Gao. 2010. Adapting boosting for information retrieval measures. Inf. Retr. 13, 3 (2010), 254-270.

[19] Yisong Yue, Rajan Patel, and Hein Roehrig. 2010. Beyond position bias: examining result attractiveness as a source of presentation bias in clickthrough data. In Proceedings of the 19th International Conference on World Wide Web, WWW 2010, Raleigh, North Carolina, USA, April 26-30, 2010. 1011-1018. 